\title{
Diversity of coupled oscillators can enhance their synchronization
}

\author{
Ghazal Montaseri ${ }^{1}$ and Michael Meyer-Hermann ${ }^{1,2}$ \\ ${ }^{1}$ Department of Systems Immunology and Braunschweig Integrated Centre of Systems Biology, Helmholtz Centre for Infection Research, \\ Braunschweig 38124, Germany \\ ${ }^{2}$ Institute for Biochemistry, Biotechnology and Bioinformatics, Technische Universität Braunschweig, Braunschweig 38106, Germany
}

(Received 23 March 2016; revised manuscript received 26 August 2016; published 18 October 2016)

\begin{abstract}
The heterogeneity of coupled oscillators is important for the degree of their synchronization. According to the classical Kuramoto model, larger heterogeneity reduces synchronization. Here, we show that in a model for coupled pancreatic $\beta$-cells, higher diversity of the cells induces higher synchrony. We find that any system of coupled oscillators that oscillates on two time scales and in which heterogeneity causes a transition from chaotic to damped oscillations on the fast time scale exhibits this property. Thus, synchronization of a subset of oscillating systems can be enhanced by increasing the heterogeneity of the system constituents.
\end{abstract}

DOI: 10.1103/PhysRevE.94.042213

\section{INTRODUCTION}

Synchronization and the emergence of collective modes are general features of interacting self-sustained oscillators. The coupling strength entrains oscillators to adjust their rhythms and to produce a pronounced collective behavior [1]. This phenomenon is widely observed in biological systems, e.g., neuronal networks [2], circadian rhythms [3], glycolytic [4] and insulin [5-8] oscillations. In this study, a general phenomenon of coupled bursting oscillators is investigated starting from the example of insulin-secreting pancreatic $\beta$-cells. Under healthy condition, $\beta$-cells respond to glucose stimulation with synchronous bursts of membrane potential oscillations $[9,10]$. These electrical bursts are associated with calcium oscillations on a slower time scale, which are required for exocytosis of insulin-carrying granules and subsequent control of blood glucose.

It was found that $\beta$-cell bursts are more robust if the cells are connected with gap junctions (connexin 36) $[11,12]$. This property was well reflected in a mathematical model of isolated versus connected $\beta$-cells, which was based on data from single ion-conduction protein activities [13]. The parameter range in which bursts of the membrane potential emerged was small in isolated as compared to connected cells [14]. Thus, electrical bursts are a fragile property of $\beta$-cells in vivo and in silico. Desynchronization of bursts between different $\beta$-cells is a hallmark of type II diabetes, such that the synchrony level among $\beta$-cells can be used to measure $\beta$-cell functionality.

Bursts of the $\beta$-cell membrane potential are initiated by a cell depolarization which is induced by inhibition of ATPdependent potassium channels (K,ATP) [15,16]. Fast spiking oscillations during each burst of the membrane potential are induced by an activation delay between voltage-gated potassium and calcium channels [10]. This introduces two time scales into the $\beta$-cell oscillations: fast spikes of the membrane potential within each burst, and a slower repetition of burst events. Oscillations on these two time scales will be distinguished in this article.

Many factors affect the synchrony of coupled oscillators, e.g., the coupling strength [17], the number of coupled oscillators [18], the network topology $[19,20]$, or the heterogeneity [21-29]. In [21], the authors reported an enhancement of synchrony in an array of Josephson junctions with disorder, which refers to the situation in which the elements of the array are not identical. The effective result of the oscillator amplitude dynamics (considered as the heterogeneity) on favoring synchronization was shown in [22]. Heterogeneous units with both amplitude and phase dynamics profit from their amplitude dynamics [which is neglected in phase (Kuramoto) oscillators] to lower the synchronization threshold. Further, studies on synchronization in networks of oscillators indirectly coupled through a medium (the so-called relay and remote synchronization) highlighted the role of heterogeneity in inducing or enhancing synchronization [23-29]. Here, we also emphasize the enhancing effect of heterogeneity on synchronization. However, the mechanism of synchrony enhancement reported here is different.

In this study, we focus on the impact of diversity on $\beta$-cell synchrony. In [14], it was shown that diversity in the density of trans-membrane proteins induces a higher robustness of the bursting oscillations of $\beta$-cells with high-frequency spikes within. In a mathematical model of coupled $\beta$-cells, diversity is reflected in a variable expression of K,ATP channels. Including diversity provides a heterogeneous population of coupled $\beta$-cells. While according to the well-known Kuramoto theory diversity among spiking oscillators is expected to disrupt their synchronization, our observations lead to an inverse result. In the model of bursting $\beta$-cells, diversity enhances synchrony. We denote the observation of diversity (D) -enhanced (E) synchrony (Syn) as DESyn. From a biological viewpoint, this means that heterogeneity improves $\beta$-cell synchronization and thus their functionality.

The goal of this paper is to unfold the phenomenon of DESyn. Toward that end, we simplify the network complexity while keeping the same structural changes in the spikes. We observe that the DESyn is independent of the network size and can still be found in a simple network containing two coupled cells. In the remaining model of two coupled cells, we further reduce the complexity in the structure of spikes employing the normal form theorem. This prepares a good framework to track the mathematical reason for the DESyn.

The paper is organized as follows. In Sec. II, first we model connected $\beta$-cells in an islet, next the heterogeneity of cells is 
TABLE I. Acronyms for the different plasma membrane proteins contributing to ion flow through the membrane. "Inward" and "outward" specify in which direction the ion flows, i.e., in or out of the cell.

\begin{tabular}{ll}
\hline \hline $\mathrm{Na}, \mathrm{K}$ & Sodium-potassium exchanger $\left(\mathrm{Na}^{+}\right.$outward, $\mathrm{K}^{+}$inward $)$ \\
$\mathrm{Na}, \mathrm{V}$ & Voltage-gated sodium channels (inward) \\
$\mathrm{NCX}$ & Sodium-calcium exchanger $\left(\mathrm{Na}^{+}\right.$inward, $\mathrm{Ca}^{2+}$ outward $)$ \\
$\mathrm{PMCA}$ & ATP-driven plasma-membrane-calcium-ATPase $\left(\mathrm{Ca} \mathrm{Ca}^{2+}\right.$ outward) \\
$\mathrm{K}, \mathrm{ATP}$ & ATP-driven (and glucose-dependent) potassium channels (outward) \\
$\mathrm{K}, \mathrm{V}$ & Voltage-gated potassium channels (delayed rectifier) (outward) \\
$\mathrm{sK}, \mathrm{Ca}$ & Small conductance calcium-gated potassium channel (outward) \\
$\mathrm{K}, \mathrm{Ca}$ & Large conductance voltage- and calcium-gated potassium channel (outward) \\
$\mathrm{Ca}, L$ & $L$-type voltage-dependent calcium channels (inward) \\
$\mathrm{Ca}, T$ & $T$-type voltage-dependent calcium channels (inward) \\
\hline
\end{tabular}

reflected in the model, and finally the $\lambda$ index to measure synchrony is introduced. The DESyn is explained in Sec. III. In Sec. IV, we employ the normal form theory to describe the DESyn. In Sec. V, we summarize our results.

\section{MATHEMATICAL FRAMEWORK}

We treat each $\beta$-cell as a biological oscillator and then investigate how diversity in oscillator parameters enhances their synchronization.

\section{A. Electrophysiological model of coupled $\beta$-cells}

We consider a network of 27 connected $\beta$-cells arranged in a block of $3 \times 9$ cells. Each cell $i$ in the network is described as

$$
\begin{aligned}
\frac{d N_{i}}{d t}= & -\frac{\xi}{F}\left(\rho_{\mathrm{Na}, \mathrm{V}} I_{\mathrm{Na}, \mathrm{V} ; i}+2 \alpha_{\mathrm{Na}, \mathrm{K}} \rho_{\mathrm{Na}, \mathrm{K}} I_{\mathrm{Na}, \mathrm{K} ; i}+\alpha_{\mathrm{NCX}} \rho_{\mathrm{NCX}} I_{\mathrm{NCX} ; i}+\frac{1}{6} \sum_{j=1}^{n} \rho_{\mathrm{gap}} I_{\mathrm{gap} ; i j}^{\mathrm{N}}+J_{\mathrm{Na}}\right), \\
\frac{d K_{i}}{d t}= & -\frac{\xi}{F}\left(\rho_{\mathrm{K}, \mathrm{ATP}} I_{\mathrm{K}, \mathrm{ATP} ; i}+\rho_{\mathrm{K}, \mathrm{V}} I_{\mathrm{K}, \mathrm{V} ; i}-\frac{4}{3} \alpha_{\mathrm{Na}, \mathrm{K}} \rho_{\mathrm{Na}, \mathrm{K}} I_{\mathrm{Na}, \mathrm{K} ; i}+\rho_{\mathrm{sK}, \mathrm{Ca}} I_{\mathrm{sK}, \mathrm{Ca} ; i}+\rho_{\mathrm{K}, \mathrm{Ca}} I_{\mathrm{K}, \mathrm{Ca} ; i}+\frac{1}{6} \sum_{j=1}^{n} \rho_{\mathrm{gap}} I_{\mathrm{gap} ; i j}^{\mathrm{K}}+J_{\mathrm{K}}\right), \\
\frac{d C_{i}}{d t}= & -\frac{\xi}{z_{\mathrm{Ca}} F\left(1+x_{\mathrm{c} ; i}\right)}\left(\rho_{\mathrm{Ca}, \mathrm{L}} I_{\mathrm{Ca}, \mathrm{L} ; i}+\rho_{\mathrm{Ca}, \mathrm{T}} I_{\mathrm{Ca}, \mathrm{T} ; i}+\rho_{\mathrm{PMCA}} I_{\mathrm{PMCA} ; i}-\frac{1}{3} z_{\mathrm{Ca}} \alpha_{\mathrm{NCX}} \rho_{\mathrm{NCX}} I_{\mathrm{NCX} ; i}+\frac{1}{6} \sum_{j=1}^{n} \rho_{\mathrm{gap}} I_{\mathrm{gap} ; i j}^{\mathrm{C}}+J_{\mathrm{Ca}}\right), \\
\frac{d V_{i}}{d t}= & -\frac{1}{C_{\mathrm{m}}}\left(\rho_{\mathrm{Na}, \mathrm{K}} I_{\mathrm{Na}, \mathrm{K} ; i}+\rho_{\mathrm{K}, \mathrm{ATP}} I_{\mathrm{K}, \mathrm{ATP} ; i}+\rho_{\mathrm{K}, \mathrm{V}} I_{\mathrm{K}, \mathrm{V} ; i}+\rho_{\mathrm{Na}, \mathrm{V}} I_{\mathrm{Na}, \mathrm{V} ; i}+\rho_{\mathrm{sK}, \mathrm{Ca}} I_{\mathrm{sK}, \mathrm{Ca} ; i}+\rho_{\mathrm{K}, \mathrm{Ca}} I_{\mathrm{K}, \mathrm{Ca} ; i}+\rho_{\mathrm{NCX}} I_{\mathrm{NCX} ; i}\right. \\
& \left.+\rho_{\mathrm{PMCA}} I_{\mathrm{PMCA} ; i}+\rho_{\mathrm{Ca}, \mathrm{L}} I_{\mathrm{Ca}, \mathrm{L} ; i}+\rho_{\mathrm{Ca}, \mathrm{T}} I_{\mathrm{Ca}, \mathrm{T} ; i}+\sum_{\text {ion }} \frac{1}{6} \sum_{j=1}^{n} \rho_{\mathrm{gap}} I_{\mathrm{gap} ; i j}^{\mathrm{ion}}+J_{\mathrm{Na}}+J_{\mathrm{K}}+J_{\mathrm{Ca}}\right) .
\end{aligned}
$$

The first three equations represent the intracellular dynamics of sodium $(\mathrm{N})$, potassium $(\mathrm{K})$, and calcium $(\mathrm{C})$ concentrations. $V$ is the plasma membrane potential. Each equation for the ion dynamics contains terms of the form $\rho_{x} I_{x}$, where $x$ specifies the type of membrane protein (listed in Table I), the constant parameter $\rho_{x}$ is the surface density of the transmembrane protein $x$, and the time-varying trans-membrane currents $I_{x}$ are derived from single protein characteristic [13]. Depending on the type of trans-membrane protein $x, I_{x}$ can be represented statically (e.g., $I_{\mathrm{Na}, \mathrm{K}}, I_{\mathrm{NCX}}$ ) or dynamically (e.g., $\left.I_{\mathrm{Na}, \mathrm{V}}, I_{\mathrm{K}, \mathrm{Ca}}\right)$. For details of the representation of $I_{x}$ and gating and/or activity properties of different membrane proteins, see Appendix A. The parameters $\alpha_{\mathrm{Na}, \mathrm{K}}$ and $\alpha_{\mathrm{NCX}}$ account for the stoichiometry of exchangers. $F$ is the Faraday constant, $\xi$ is the surface to volume factor, and $z_{\mathrm{Ca}}$ is the valance of the calcium ions. In the dynamics of calcium, $x_{\mathrm{c}}=\frac{c_{0} K_{\mathrm{c}}}{\left(C+K_{\mathrm{c}}\right)^{2}}$ is the calcium buffer and $C_{\mathrm{m}}$ is the plasma membrane capacitance.

In addition to the trans-membrane currents $I_{x}$ in each cell, there are two other types of currents. $J_{\text {ion }}$ and $I_{\text {gap } ; i j}^{\text {ion }}$ (ion $=\mathrm{N}, \mathrm{K}, \mathrm{C}$ ) represent the constant leakage and the dynamic gap junction currents, respectively. The different $\beta$-cells communicate with each other via the gap junction protein connexin 36. $n$ in the gap junction terms stands for the number of connected neighbor cells. The gap junction current between cell $i$ and its neighbor cell $j$ is described as

$$
I_{\text {gap } ; i j}^{\text {ion }}=\overline{g_{\text {gap }}} V_{\text {gap } ; i j}^{\text {ion }},
$$


with

$$
\frac{d V_{\mathrm{gap} ; i j}^{\mathrm{ion}}}{d t}=\frac{\overline{V_{\mathrm{gap} ; i j}^{\mathrm{ion}}}-V_{\mathrm{gap} ; i j}^{\mathrm{ion}}}{\tau_{\mathrm{gap}}^{\text {ion }}} .
$$

The asymptotic potential difference is

$$
\overline{V_{\text {gap } ; i j}^{\text {ion }}}=V_{i}-V_{j}-\tilde{V}_{i j}^{\text {ion }},
$$

where the latter term represents the Nernst potential,

$$
\tilde{V}_{i j}^{\text {ion }}=\frac{R T}{z_{x} F} \ln \left(\frac{\text { ion }_{j}}{\operatorname{ion}_{i}}\right),
$$

with the Rydberg (molar) gas constant $R=8.315 \mathrm{~J} /(\mathrm{K} \mathrm{mol})$, the temperature $T$, and ion $_{i(j)}$ representing the concentrations of the respective ion in both cells.

The model parameters are taken from [13,14] with 10 gap junctions per cell-cell contact. The cells are stimulated with the physiological level of $\gamma=11 \mathrm{mM}$ glucose. Note that synchronization may occur between low-frequency (bursts) and/or high-frequency (spikes within bursts) components. Synchronization of the low-frequency components is tightly connected to the number of gap junctions per cell to cell contact. The higher the number of gap junctions per contact, the higher is the synchrony of the low-frequency components. However, stochastic variation in the gap junction currents does not have a major effect on $\beta$-cell activity [14]. In this study, the number of gap junctions is chosen large enough to entrain the low-frequency components, and we focus mainly on the behavior of spikes within bursts.

\section{B. Introducing heterogeneity in the $\beta$-cell model}

To include heterogeneity in the model, we varied the expression level of the burst initiating ion-conducting transmembrane protein $\rho_{\mathrm{K} \text {, ATP }}$ among the $\beta$-cells by sampling from a Gaussian distribution with standard deviation $\sigma$ reflecting cell diversity. Here, we mainly focus on ATP-dependent potassium channels because initiation of $\beta$-cell response to elevated glucose level is handled through these channels. In addition, findings of [14] reveal that stochasticity in the $\rho_{\mathrm{K}, \text { ATP }}$ parameter improves functionality of $\beta$-cells. This motivates us to highlight this parameter and look for a mathematical reason for this observation.

\section{Synchrony index $\lambda$}

The synchrony index $\lambda$ compares the membrane potentials of connected $\beta$-cells and quantifies the concept of synchronization to a number in $[0,1] ; 1$ denotes the highest synchrony level, while 0 refers to complete asynchrony [30]. This index is based on a stroboscopic approach, i.e., we observe the phase of the second oscillator $\phi_{2}$ whenever the phase of the first oscillator $\phi_{1}$ reaches a fixed value $\Theta$ (phase stroboscope). This can be formulated as

$$
\psi=\left.\phi_{2} \bmod 2 \pi\right|_{\phi_{1} \bmod 2 \pi=\Theta} .
$$

Instantaneous phases of signals to be compared-here, time courses of the $\beta$-cell membrane potential_can be calculated from the Hilbert transform of the processed (i.e., removal of linear trends) signal. First, the interval $[0,2 \pi]$ is divided into $N$ bins, and the phase of the first oscillator is binned accordingly.
We denote $\hat{\phi}_{l}$ as the values of $\phi_{1} \bmod 2 \pi$ falling into the $l$ th bin and $M_{l}$ as the number of points within this bin. Then, with the help of (6), we compute $M_{l}$ corresponding values $\psi_{j, l}\left(j=1,2, \ldots, M_{l}\right)$. If the two oscillators are synchronized, $\psi_{j, l}$ values group around a value and their distribution displays a peak. If oscillators are not synchronized, $\psi_{j, l}$ values are uniformly distributed on the interval $[0,2 \pi]$. This is quantified by the index

$$
\Lambda_{l}=M_{l}^{-1} \sum_{j=1}^{M_{l}} e^{\psi_{j, l}} .
$$

$\left|\Lambda_{l}\right|=1$ denotes complete dependence between both phases, while small values of $\left|\Lambda_{l}\right|$ correspond to no dependence. Finally, averaging over all $N$ bins gives the synchrony index,

$$
\lambda=N^{-1} \sum_{l=1}^{N}\left|\Lambda_{l}\right| .
$$

For more than two oscillators, $\lambda$ is obtained as the average of synchrony indices for each pair of oscillators [31]. More details about the mathematical basis of $\lambda$ and the pseudocode of $\lambda$ calculation can be found in [30] and [32], respectively.

Among all synchrony indices introduced in [30], here we selected the one that is on the one hand established for real data with complicated time series, and on the other hand sensitive to both low- and high-frequency components. In fact, all frequencies contribute to the instantaneous phase and, consequently, to $\lambda$. While synchronization of bursts is experimentally reported to be directly related to more insulin release [5], our theoretical results (Fig. 3) highlight the importance of the suppression of spikes for higher calcium concentrations and thus insulin release. Since $\lambda$ captures changes in the phases of both spikes and bursts, it is a good candidate to measure the impact of diversity on the insulin release.

\section{WHAT IS DESyn?}

Assuming a network of coupled heterogeneous $\beta$-cells, we vary the variance of $\rho_{\mathrm{K} \text {,ATP }}$ and measure the synchrony index $\lambda$. For small values of $\sigma, \lambda$ adopts small values (with the minimum value at $\sigma=0$ and for different initial conditions) and becomes larger with increasing $\sigma$ [Fig. 1(a)]. Thus, a higher diversity (D) $\sigma$ enhances (E) synchrony (Syn) $\lambda$. According to the well-known Kuramoto theory, a decreasing curve $\lambda(\sigma)$ would have been expected. We denote this counterintuitive result as DESyn.

To understand DESyn, we analyze the $\beta$-cell membrane potential for two selected diversity levels: $\sigma=0.002$ (low diversity) and $\sigma=0.04$ (high diversity). For low diversity $(\sigma=0.002)$ the coupling with gap junctions induces chaotic oscillations [Fig. 2(a)]. The gap junction current synchronizes the slow bursts, but the spikes within the bursts are asynchronous (i.e., with no fixed phase relation) [see the zoomed view in Fig. 2(b)]. Spikes and bursts can both become synchronized by increasing coupling strength (result not shown).

For large diversity $(\sigma=0.04)$, the chaotic oscillations observed at lower diversity disappear [Fig. 2(c)]. Spikes within 

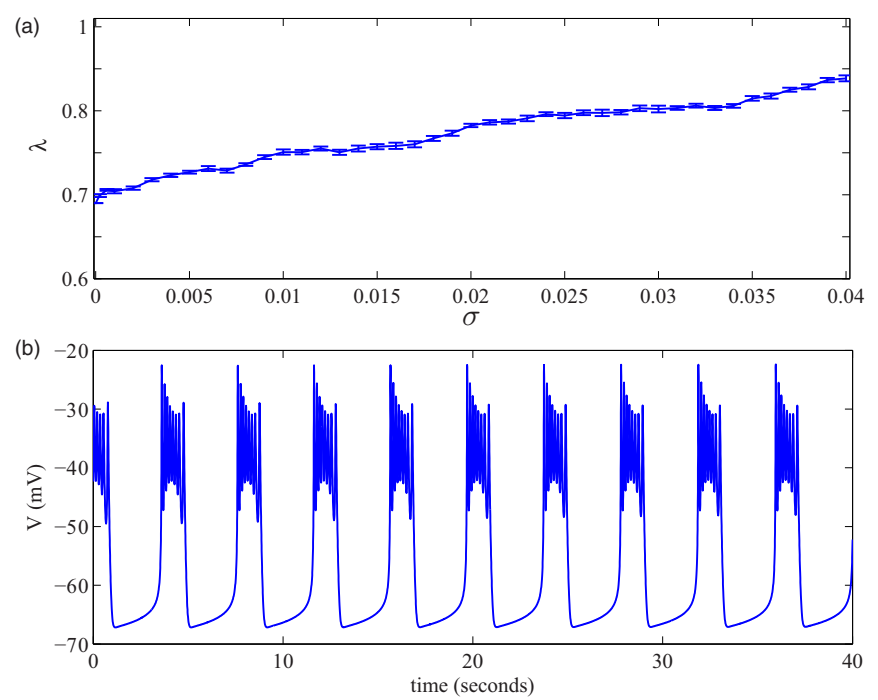

FIG. 1. (a) Synchrony index $\lambda$ for different cell diversities. (b) Time courses of a typical $\beta$-cell membrane potential $V$ in a homogeneous network, i.e., with identical cells.

the bursts are damped, and during each burst a spike-free plateau is observed. The disappearance of oscillations was also observed in Stuart-Landau oscillators coupled with sufficiently large diversity and coupling strength [33,34]. These pulled each other off their limit cycles and collapsed into the origin
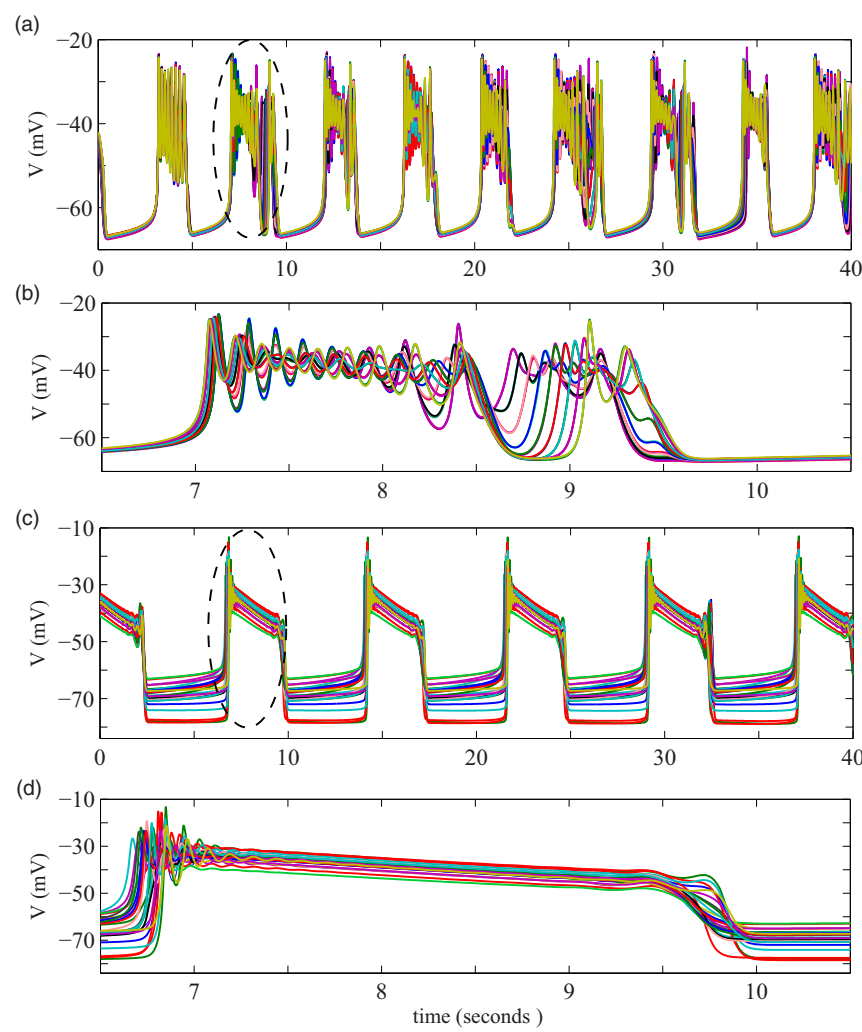

FIG. 2. Time courses of membrane potential $V$ with each gray shade (color) representing one $\beta$-cell. (a) Low diversity $(\sigma=0.002$ ), (c) high diversity $(\sigma=0.04)$, and (b) and (d) zoomed view of the region depicted by the dashed line in (a) and (c), respectively.
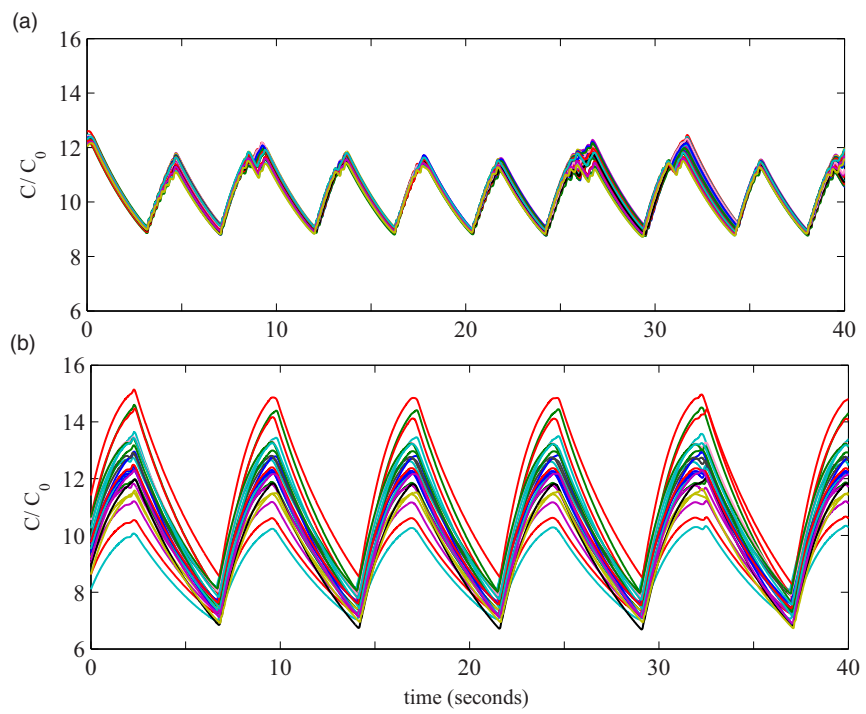

FIG. 3. Time courses of intracellular calcium $\mathrm{C}$ corresponding to Fig. 2 with each gray shade (color) representing one $\beta$-cell for (a) low $(\sigma=0.002)$ and (b) high diversity $(\sigma=0.04)$. Intracellular calcium is plotted as a ratio to the resting concentration of $C_{0}=0.1 \mu \mathrm{M}$.

(amplitude death). For the $\beta$-cells, amplitude death occurs in the spikes only. They do not collapse into the origin because the oscillations are induced on top of the bursts.

Figure 2(b) exhibits chaotic as well as asynchronous oscillations, which are lacking in Fig. 2(d). Chaotic and/or asynchronous oscillations are responsible for the reduction of overall synchrony. This implies that the DESyn is related to structural changes in the behavior of spikes within the bursts. Next, we asked why these structural changes are induced by variation of the diversity $\sigma$.

In the transition from low diversity to high diversity, via a Hopf bifurcation, the limit cycle leading to spikes loses its stability, resulting in a spike-free plateau. Due to the complexity in the $\beta$-cell model, analytic expression of the Hopf bifurcation point in terms of parameters is impossible. However, numerical simulations estimate the Hopf bifurcation point at $\sigma^{*}=0.035$.

As mentioned before, insulin is released in response to glucose-driven electrical stimulation, which increases the intracellular calcium concentration and subsequent exocytosis of insulin-carrying granules. Thus, monitoring the calcium concentration is a good candidate to track the impact of the diversity of ion-conducting trans-membrane proteins on the released insulin. In Figs. 1 and 2, we focused on the response of the $\beta$-cell membrane potential because a variation of membrane potential causes changes in the calcium dynamics and, thus, release of insulin (on a different time scale). In Fig. 3, we plot the intracellular calcium concentrations of the $\beta$-cells (divided to the resting concentration of $0.1 \mu \mathrm{M}$ ) for low $(\sigma=0.002)$ and high $(\sigma=0.04)$ diversities (corresponding to Fig. 2).

In Fig. 3(a), for low diversity the calcium concentration oscillates with smaller amplitude, indicating a lower calcium concentration and, thus, less exocytosis of insulin-carrying granules. In addition, chaotic and asynchronous oscillations of the membrane potentials are reflected in the malformed (not 
sharp) triangular peak of the calcium waveform. Therefore, increasing diversity has two impacts on the calcium oscillations. One is an increase in the amplitude of calcium concentration, and the other is induction of a more physiological triangular waveform with a sharp peak. The used synchrony index quantifies the impact of diversity on the collective behavior of membrane potentials in the coupled $\beta$-cells. The collective behavior of $\beta$-cells affects the calcium concentration, which is in turn related to insulin release. Thus, DESyn elevates calcium concentration and consequently insulin release. Note that, although for both low and high diversities bursts of membrane potentials are synchronized [Figs. 2(a) and 2(c)], elevated calcium concentrations in Fig. 3(b) highlight the effective role of spikes dynamics on the proper $\beta$-cell functionality.

\section{NORMAL FORM ANALYSIS}

We simplified the system as much as possible while keeping the same structural changes in the spikes. It turned out that the structural changes associated with the DESyn are independent of the network size and can still be observed in a simple network of two diffusively coupled oscillators,

$$
\begin{aligned}
& \dot{X}_{1}=\frac{d X_{1}}{d t}=F\left(X_{1} ; \kappa_{1}\right)+g_{c} D\left(X_{2}-X_{1}\right), \\
& \dot{X}_{2}=\frac{d X_{2}}{d t}=F\left(X_{2} ; \kappa_{2}\right)+g_{c} D\left(X_{1}-X_{2}\right),
\end{aligned}
$$

where $\quad X_{j}=\left[\begin{array}{llll}X_{j 1} & X_{j 2} & \cdots & X_{j n}\end{array}\right] \in \mathbb{R}^{n}, j=1,2, \quad F=$ $\left[\begin{array}{llll}F_{1} & F_{2} & \cdots & F_{n}\end{array}\right] \in \mathbb{R}^{n}$, and $\kappa_{j} \in \mathbb{R}$. The term $g_{c} D$ represents the coupling with the coupling strength $g_{c}$ and the $n \times n$ coupling matrix $D$. Details of mapping (1)-(5) to (9) can be found in Appendix B. Due to the complexity of the $\beta$-cell model (1)-(5), involving chaotic spikes within bursts, other factors may lead to spike suppression. For instance, chaos itself may be considered as a source of dynamic diversity. In this study, we focus on the impact of parameter diversity on spike suppression.

To further simplify the analysis by application of the "normal form" theorem, the normal form of (9) is derived [35,36]. It is assumed that for $g_{c}=0$ and at the bifurcation point $\kappa_{j}=\kappa^{*}$ the system (9) admits a supercritical Hopf bifurcation at which the stable equilibrium points of (9) lose their stability when the growing $\kappa_{j}$ values cross $\kappa^{*}$.

In addition, we assume the following:

(i) The oscillators (9) are near the Hopf bifurcation, i.e., with $0<\varepsilon \ll 1$ and $\mu_{j}=O(1)$,

$$
\kappa_{j}-\kappa^{*}=\varepsilon^{2} \mu_{j}, \quad j=1,2 .
$$

(ii) The coupling strength $g_{c}$ is in the same order of magnitude as the deviation from criticality, i.e., with $v=$ $O(1)$,

$$
g_{c}=\varepsilon^{2} v .
$$

The weakly nonlinear oscillators (9) are expanded in a Taylor series around the steady-state solution $\bar{X}_{j}$. With the vectors $Y_{j}=X_{j}-\bar{X}_{j}$, we get

$$
\begin{aligned}
\dot{Y}_{j}= & L\left(\kappa_{j}\right) Y_{j}+M\left(\kappa_{j}\right) Y_{j} Y_{j}+N\left(\kappa_{j}\right) Y_{j} Y_{j} Y_{j}+\cdots \\
& +g_{c} D\left(Y_{k}-Y_{j}\right), \quad j, k=1,2, \quad j \neq k,
\end{aligned}
$$

where $L\left(\kappa_{j}\right)$ is the Jacobian matrix of $F\left(X_{j} ; \kappa_{j}\right)$ calculated at $X_{j}=\bar{X}_{j}$. The nonlinear terms in (12) are vectors with the $m$ th $(1 \leqslant m \leqslant n)$ component given by

$$
\begin{aligned}
\left(M Y_{j} Y_{j}\right)_{m} & =\frac{1}{2 !} \sum_{p=1}^{n} \sum_{q=1}^{n} \frac{\partial^{2} F_{m}\left(\bar{X}_{j} ; \kappa_{j}\right)}{\partial X_{j p} \partial X_{j q}} Y_{j p} Y_{j q}, \\
\left(N Y_{j} Y_{j} Y_{j}\right)_{m} & =\frac{1}{3 !} \sum_{p=1}^{n} \sum_{q=1}^{n} \sum_{s=1}^{n} \frac{\partial^{3} F_{m}\left(\bar{X}_{j} ; \kappa_{j}\right)}{\partial X_{j p} \partial X_{j q} \partial X_{j s}} Y_{j p} Y_{j q} Y_{j s} .
\end{aligned}
$$

Using the fact that at the bifurcation point $\kappa^{*}$ the Jacobian matrix $L$ has a pair of purely imaginary eigenvalues $\pm i \omega_{0}$, expanding $L, M$, and $N$ around $\kappa^{*}$, and using the assumptions (10) and (11), (12) is reformulated as

$$
\begin{aligned}
\dot{Y}_{j}= & \left(L_{0}+\varepsilon^{2} \mu_{j} \tilde{L}\right) Y_{j}+M_{0} Y_{j} Y_{j}+N_{0} Y_{j} Y_{j} Y_{j} \\
& +\cdots+\varepsilon^{2} v D\left(Y_{k}-Y_{j}\right), \quad j, k=1,2, \quad j \neq k,
\end{aligned}
$$

where $\tilde{L}=d L\left(\kappa^{*}\right) / d \kappa$, and $L_{0} Y_{j}, M_{0} Y_{j} Y_{j}$, and $N_{0} Y_{j} Y_{j} Y_{j}$ are $L, M Y_{j} Y_{j}$, and $N Y_{j} Y_{j} Y_{j}$ calculated at $\kappa_{j}=\kappa^{*}$. We look for a solution in the form

$$
Y_{j}(t)=\varepsilon G_{j}(t, \tau)+\varepsilon^{2} H_{j}(t, \tau)+\cdots,
$$

with $\tau=\varepsilon^{2} t$. The solution can be calculated by substituting (15) in (14), setting equal the coefficients of different powers of $\varepsilon$, and successively solving the resulting set of equations (these steps are done in Appendix C). For the complex oscillation amplitude $A_{j}$ of each oscillator, this yields the evolution equations

$$
\begin{aligned}
\frac{d A_{j}}{d \tau}= & \mu_{j}(a+i b) A_{j}-(c+i d)\left|A_{j}\right|^{2} A_{j} \\
& +v(e+i f)\left(A_{k}-A_{j}\right), \quad j, k=1,2, \quad j \neq k,
\end{aligned}
$$

where the real constants $a, b, c, d, e$, and $f$ are defined as

$$
\begin{aligned}
a+i b & =U^{*} \tilde{L} U \\
c+i d & =-2 U^{*} M_{0} U V_{o}-2 U^{*} M_{0} \bar{U} V_{+}-3 U^{*} N_{0} U U \bar{U} \\
e+i f & =U^{*} D U .
\end{aligned}
$$

Here, $U$ (with the complex conjugate $\bar{U}$ ) and $U^{*}$ (with the complex conjugate $\bar{U}^{*}$ ) are the right and left eigenvectors of $L_{0}$ at their eigenvalues $i \omega_{o}$, respectively. These vectors are normalized, i.e., $U^{*} U=\bar{U}^{*} \bar{U}=1$. Further,

$$
\begin{aligned}
& V_{+}=\bar{V}_{-}=-\left(L_{0}-2 i \omega_{o} I\right)^{-1} M_{0} U U, \\
& V_{0}=-2 L_{0}^{-1} M_{0} U \bar{U} .
\end{aligned}
$$

Defining

$$
A_{j}(\tau)=\sqrt{\frac{\mu_{j} a}{c}} r_{j}(\tau) e^{i \theta_{j}(\tau)}
$$

and the phase difference $\theta=\theta_{2}-\theta_{1}$, (16) is reformulated in polar coordinates, yielding

$$
\begin{aligned}
& \dot{r}_{1}=r_{1}\left(1-r_{1}^{2}\right)+\Gamma \sqrt{\Delta}(\cos \theta-\alpha \sin \theta) r_{2}-\Gamma r_{1}, \\
& \dot{r}_{2}=\Delta r_{2}\left(1-r_{2}^{2}\right)+\frac{\Gamma}{\sqrt{\Delta}}(\cos \theta+\alpha \sin \theta) r_{1}-\Gamma r_{2},
\end{aligned}
$$




$$
\begin{aligned}
\dot{\theta}= & \beta(\Delta-1)+\eta\left(r_{1}^{2}-\Delta r_{2}^{2}\right)-\frac{\Gamma}{\sqrt{\Delta}}(\sin \theta-\alpha \sin \theta) \frac{r_{1}}{r_{2}} \\
& -\Gamma \sqrt{\Delta}(\sin \theta+\alpha \cos \theta) \frac{r_{2}}{r_{1}},
\end{aligned}
$$

where $r_{1,2}$ are oscillation amplitudes, $\tau$ is rescaled to $\hat{\tau}=$ $\mu_{1} a \tau$, and

$$
\alpha=\frac{f}{e}, \quad \beta=\frac{b}{a}, \quad \eta=\frac{d}{c}, \quad \Gamma=\frac{v e}{\mu_{1} a}, \quad \Delta=\frac{\mu_{2}}{\mu_{1}} .
$$

Under the assumptions (10) and (11), (9) is locally topologically equivalent to the normal form (20).

In the normal form (20), $\alpha, \beta$, and $\eta$ are predetermined parameters based on $F\left(X_{j} ; \kappa_{j}\right), j=1,2$, and the coupling matrix $D$ in (9). Note that the coupling strength plays no role in these parameters [see (17) and (21)]. Finding analytical expressions that relate this set of parameters to the parameters of the $\beta$-cell model (1) [or, in general, to the parameters of model (9)] is not possible. However, the values of these parameters can be determined using numerical algorithms. ${ }^{1}$ To reduce the complexity, we use the minimal model of $\beta$-cells [37], which still exhibits DESyn. The algorithm starts with calculation of the steady state and location of Hopf bifurcation (in the fast subsystem). Next, left and right eigenvectors are obtained. Finally, these data can be used in (17), (18), and (21) to have $\alpha=-0.52, \beta=-23.55$, and $\eta=15.55$.

The remaining two parameters $\Gamma$ and $\Delta$ are free parameters. $\Delta$ measures the relative difference between the two oscillators in terms of their distances to the Hopf bifurcation point. $\Gamma$ represents the coupling strength. At $\Delta=1$, both oscillators are identical, i.e., homogeneity. $\Delta$ close to 1 represents small diversity, and as $\Delta$ increasingly deviates from 1 , diversity increases. In this relationship, the strength of the gap junction coupling and the diversity of $\rho_{\mathrm{K} \text {,ATP }}(\sigma)$ in the $\beta$-cell model correspond to $\Gamma$ and the deviation of $\Delta$ from 1, respectively.

From a dynamical system point of view, the existence and stability of steady states of (20) depend on the values of the free parameters $\Delta$ and $\Gamma$. For fixed values of $\Gamma(\Gamma=1, \ldots, 5)$, in Fig. 4, we plot steady-state solutions of $\dot{r}_{j}=0, j=1,2$ as $\Delta$ varies. In each curve, solid and dotted lines correspond to stable and unstable steady-state solutions, respectively. Variation of $\Delta$ changes not only the number of steady-state solutions but also the stability of the solutions. Moving along the $\Delta$ axis [Fig. 4(a), $\Gamma=1$ ], in the beginning only one stable steady state exists. Around $\Delta=0.88$, two unstable branches of solutions appear, one of which becomes stable near $\Delta=0.97$ (bistability). At higher values of $\Delta$, the middle branch loses its stability and one stable and two unstable steady states emerge. Finally, the normal form model (20) shows only one stable steady state.

In the $\beta$-cell model, spikes within the bursts are due to the emergence of a Hopf bifurcation in the fast dynamics. The observations in Fig. 2 have reduced the study of the DESyn to the investigation of behaviors of spikes within the bursts. As the reduced model (20) can undergo Hopf bifurcations leading

\footnotetext{
${ }^{1}$ For details of parameter estimation, see http://mrb.niddk.nih.gov/sherman/MathCoup.html
}
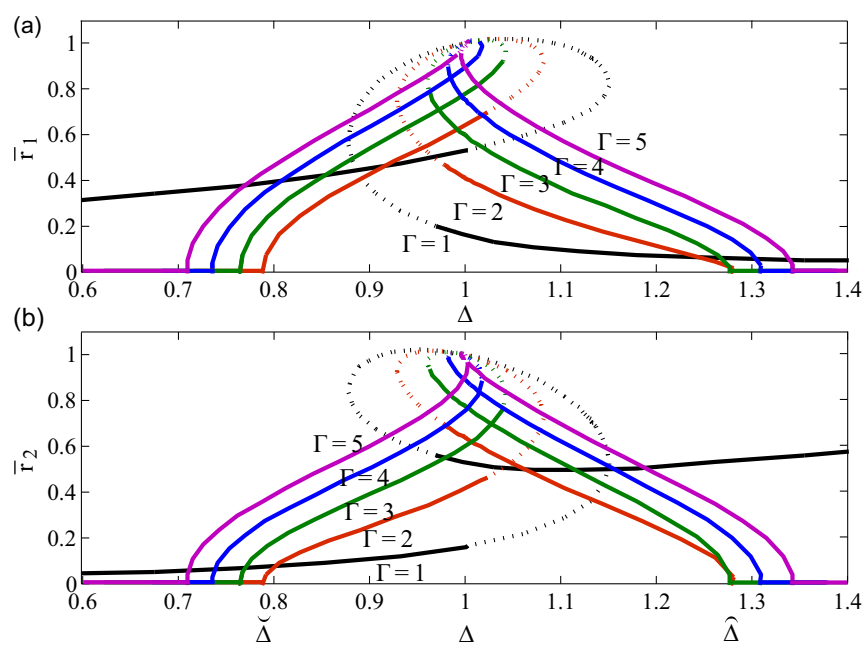

FIG. 4. Steady-state solutions of $r_{1}$ (a) and $r_{2}$ (b) as $\Delta$ changes for different values of $\Gamma$. Each gray shade (color) refers to one value of $\Gamma=1, \ldots, 5$. Solid and dotted lines correspond to stable and unstable steady-state solutions, respectively. For each $\Gamma$, depending on the value of $\Delta$, the reduced model (20) may have a unique stable steady state or show bistability (i.e., the existence of two stable steady states). Note that, for each $\Gamma$, flipping the $\bar{r}_{1}$ curve at the line $\Delta=1$ gives the corresponding curve of $\bar{r}_{2}$ and vice versa.

to the onset or fading of spikes, it provides a simple framework for the analysis of different behaviors of the spikes in the $\beta$-cell model. Thereby, the results from the reduced model (20) can be interpreted in terms of the $\beta$-cell model:

(i) Existence and stability of the steady-state solutions $\bar{r}_{j}=$ 0 of (20) means the absence of oscillations in the reduced model, which corresponds to the death of spike amplitudes during bursts in the $\beta$-cell model.

(ii) If no stable steady-state solution of the equation $\dot{r}_{j}=0$ exists, the reduced model may show quasiperiodic
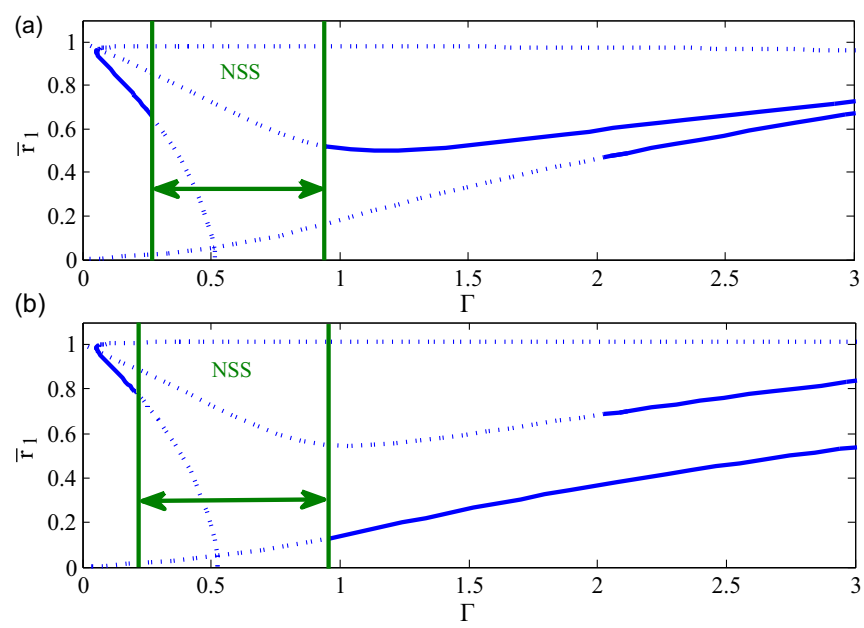

FIG. 5. Steady-state solutions of $r_{1}$ as $\Gamma$ changes for $\Delta=0.98$ (a) and $\Delta=1.02$ (b). Solid and dotted lines correspond to stable and unstable steady-state solutions, respectively. The vertical lines delineate a region of $\Gamma$ values with NSSs. The results for $\bar{r}_{2}$ are the same. 
(QP) or phase-trapped (PT) oscillations (depending on the $\theta$ dynamics), which we will summarize here as nonstationary solutions (NSSs) and which correspond to irregular spikes within bursts in the $\beta$-cell model.

As can been seen from the solutions of $\dot{r}_{j}=0$ against $\Delta$ for different different $\Gamma$ (Fig. 4), spike death $\left(\bar{r}_{j}=0\right)$ emerges when $\Delta$ diverges from 1 . This means that, for sufficiently large $\Gamma>1.9, \breve{\Delta}(\Gamma)$ and $\widehat{\Delta}(\Gamma)$ exist such that for $\Delta<\breve{\Delta}(\Gamma)$ or $\Delta>\widehat{\Delta}(\Gamma)$ stable steady-state solutions $\bar{r}_{1,2}=0$, i.e., no oscillations, are found. We conclude that spike death observed in the $\beta$-cell model [Figs. 2(c) and 2(d)] occurs for diversities above a critical value.

For small diversity, i.e., for $\Delta$ near identity, NSSs of $\dot{r}_{j}=0$ appear in a particular range of $\Gamma$ values (vertical lines in Fig. 5). Thus, the irregular behavior of spikes in the $\beta$-cell model [Figs. 2(a) and 2(b)] is recovered here in the case of small diversity of the two oscillators in the reduced model.

\section{CONCLUSION}

Synchronization of electrical activity in coupled pancreatic $\beta$-cells precedes the normal release of insulin and is disrupted in diabetic patients. In this study, we investigated the role of cell diversity (modeled as variable expression of ATP-dependent potassium channels) on the synchrony level among connected $\beta$-cells. If we treat each $\beta$-cell as a biological oscillator, an increased heterogeneity level enhances synchronization of the coupled $\beta$-cells. The normal form theory provides a suitable framework to disentangle this observation and solve the intuitive contradiction with the well-known Kuramoto theory.

Motivated by the observed DESyn in the heterogeneous ensemble of $\beta$-cells and using the normal form theory, we found that the DESyn requires two separated time scales, realized here as bursts and spikes. The analysis of single bursts revealed that the effect is based on structural properties of the spikes within the bursts. For that reason, theories that rely on phase oscillators, such as the traditional Kuramoto model, cannot capture this effect.

The normal form analysis showed that the DESyn is a general feature of any set of bursting oscillators in which diversity controls a soft switch between bursts with chaotic spikes and spike-free bursts. Here, the mechanism underlying DESyn is the suppression of spikes within bursts by the diversity of the oscillators. Instead of diversity, in some oscillator models (e.g., in the model of coupled HindmarshRose neurons with chaotic bursting oscillations), increasing the synaptic coupling strength leads to spike suppression.

In addition to DESyn, other mechanisms for diversityenhanced synchronization were reported, e.g., in the phenomenon of relay and remote synchronization. These types of synchronization both refer to a collective state, in which uncoupled oscillators synchronize through the action of intermediate oscillators but do not synchronize with them. In remote synchronization, heterogeneity is reflected in the amplitude dynamics of oscillators whose modulation permits transition of information for synchronization [27,28]. Heterogeneity in the relay synchronization is introduced by adding mismatched nodes that lower the threshold for synchronization [24-26].
Thus, lowering the synchronization threshold and modulation of amplitude dynamics are two other mechanism leading to higher synchrony. Another potential mechanism for the diversity-enhanced synchronization is the case when the coupling strength between oscillators is inversely proportional to the mean field.

Instead of normal form analysis, another interesting attempt is to approximate the solutions in parametrized forms using an ansatz method with two general and detuned oscillators (basis functions) and two different time scales. This approach may be investigated in our future work.

In summary, a subset of systems exist, for which synchronization can be enhanced by increasing the heterogeneity of the constituents of the system, as realized, for example, in glucose-controlling $\beta$-cells coupled in pancreatic islets. This exciting result has major implications for our understanding of insulin release, and it paves the way for new intervention strategies.

\section{ACKNOWLEDGMENTS}

We thank Sebastian Binder for revising the manuscript. M.M.H. was supported by the Helmholtz Association crossprogram activity Metabolic Dysfunction and Human Disease.

\section{APPENDIX A: CONDUCTANCE OF MEMBRANE PROTEINS}

The $I_{x}$ currents entering $\beta$-cell model (1) are themselves dynamic or static quantities depending on the membrane proteins $x$. In the following, the gating and/or activity properties of different membrane proteins are described. The index $i$ in (1) enumerating the considered cell was omitted here. The activation dynamics of active carrier proteins relies on the Hill function,

$$
H\left(x, x_{\mathrm{h}}, n\right)=\frac{x^{n}}{x^{n}+x_{\mathrm{h}}^{n}},
$$

where $x$ denotes some concentration, $x_{\mathrm{h}}$ is the corresponding concentration of half-maximal activation, and $n$ is the Hill coefficient. The asymptotic state of activation and inactivation is described by two functions of sigmoidal shape,

$$
\begin{aligned}
\sigma_{\text {act }}\left(x, x_{\mathrm{h}}, x_{\mathrm{h} 0}\right) & =\frac{1}{1+\exp \left\{\left(x_{\mathrm{h}}-x\right) / x_{\mathrm{h} 0}\right\}}, \\
\sigma_{\text {inact }}\left(x, x_{\mathrm{h}}, \bar{x}_{\mathrm{h} 0}\right) & =\frac{1}{1+\exp \left\{\left(x-x_{\mathrm{h}}\right) / \bar{x}_{\mathrm{h} 0}\right\}},
\end{aligned}
$$

where $x_{\mathrm{h}}$ is the concentration of the half-maximal value, and $x_{\mathrm{h} 0}$ and $\bar{x}_{\mathrm{h} 0}$ regulate the activation and inactivation steepness, respectively.

$I_{\mathrm{Na}, \mathrm{K}}$ (the sodium-potassium exchanger), which transports sodium out of the cell and potassium into the cell, is modeled as

$$
I_{\mathrm{Na}, \mathrm{K}}=\overline{I_{\mathrm{Na}, \mathrm{K}}}\left[1-H\left(K, K_{\mathrm{Na}, \mathrm{K}}, n_{\mathrm{Na}, \mathrm{K}}\right)\right] H\left(N, \tilde{K}_{\mathrm{Na}, \mathrm{K}}, \tilde{n}_{\mathrm{Na}, \mathrm{K}}\right),
$$

where $\overline{I_{\mathrm{Na}, \mathrm{K}}}$ denotes the maximum current.

The voltage-gated sodium channel $(\mathrm{Na}, \mathrm{V})$ opens when the cell is depolarized. $I_{\mathrm{Na}, \mathrm{V}}$ is composed of two factors, one 
describing the opening probability of the channel, the other describing the flow through the open channel,

$$
I_{\mathrm{Na}, \mathrm{V}}=h_{\mathrm{Na}, \mathrm{V}} g_{\mathrm{Na}, \mathrm{V}} \overline{g_{\mathrm{Na}, \mathrm{V}}}\left(V-\overline{V_{\mathrm{Na}}}\right) .
$$

$\overline{V_{\mathrm{Na}}}$ is the sodium reversal potential and $\overline{g_{\mathrm{Na}, \mathrm{V}}}$ is the maximum conductance. The opening and inactivation probabilities $g_{\mathrm{Na}, \mathrm{V}}$, $h_{\mathrm{Na}, \mathrm{V}}$ are assumed to exponentially reach their asymptotic values and, thus, follow the kinetic equations

$$
\begin{aligned}
& \frac{d g_{\mathrm{Na}, \mathrm{V}}}{d t}=\frac{\sigma_{\mathrm{act}}\left(V, V_{\mathrm{Na}, \mathrm{V}}, \kappa_{\mathrm{Na}, \mathrm{V}}\right)-g_{\mathrm{Na}, \mathrm{V}}}{\tau_{\mathrm{Na}, \mathrm{V}}}, \\
& \frac{d h_{\mathrm{Na}, \mathrm{V}}}{d t}=\frac{\sigma_{\text {inact }}\left(V, W_{\mathrm{Na}, \mathrm{V}}, \lambda_{\mathrm{Na}, \mathrm{V}}\right)-h_{\mathrm{Na}, \mathrm{V}}}{\theta_{\mathrm{Na}, \mathrm{V}}} .
\end{aligned}
$$

The sodium-calcium exchanger (NCX) takes advantage of the electrochemical gradient of sodium (pushing sodium into the cell) to transport calcium ions against its electrochemical gradient. The current is derived from a corresponding Hill function

$$
I_{\mathrm{NCX}}=\overline{I_{\mathrm{NCX}}} H\left(C, C_{\mathrm{NCX}}, n_{\mathrm{NCX}}\right) .
$$

PMCA is an ATP-driven calcium pump extruding calcium from the cell to the extracellular medium, which is modeled as

$$
I_{\mathrm{PMCA}}=\overline{I_{\mathrm{PMCA}}} H\left(C, C_{\mathrm{PMCA}}, n_{\mathrm{PMCA}}\right),
$$

where $\overline{I_{\mathrm{PMCA}}}$ is the maximum current.

The ATP-driven potassium channel uses ATP to open the channel and to release potassium from the cell. The single protein current is modeled by

$$
\begin{aligned}
I_{\mathrm{K}, \mathrm{ATP}} & =\left(1-g_{\mathrm{K}, \mathrm{ATP}}\right) \overline{g_{\mathrm{K}, \mathrm{ATP}}}\left(V-\overline{V_{\mathrm{K}}}\right), \\
\frac{d g_{\mathrm{K}, \mathrm{ATP}}}{d t} & =\frac{\sigma_{\mathrm{act}}\left(\gamma, \gamma_{\mathrm{K}, \mathrm{ATP}}, \kappa_{\mathrm{K}, \mathrm{ATP}}\right)-g_{\mathrm{K}, \mathrm{ATP}}}{\tau_{\mathrm{K}, \mathrm{ATP}}} .
\end{aligned}
$$

Here, $\gamma$ is the glucose concentration. All other parameters are in full analogy to the voltage-gated sodium channel $\mathrm{Na}, \mathrm{V}$.

The voltage-gated potassium channel $(\mathrm{K}, \mathrm{V})$ increases the potassium efflux from the cell once the cell is depolarized. The model for this channel is in complete analogy to the voltagegated sodium channel (A4)

$$
\begin{aligned}
I_{\mathrm{K}, \mathrm{V}} & =h_{\mathrm{K}, \mathrm{V}} g_{\mathrm{K}, \mathrm{V}} \overline{g_{\mathrm{K}, \mathrm{V}}}\left(V-\overline{V_{\mathrm{K}}}\right), \\
\frac{d g_{\mathrm{K}, \mathrm{V}}}{d t} & =\frac{\sigma_{\text {act }}\left(V, V_{\mathrm{K}, \mathrm{V}}, \kappa_{\mathrm{K}, \mathrm{V}}\right)-g_{\mathrm{K}, \mathrm{V}}}{\tau_{\mathrm{K}, \mathrm{V}}}, \\
\frac{d h_{\mathrm{K}, \mathrm{V}}}{d t} & =\frac{\sigma_{\text {inact }}\left(V, W_{\mathrm{K}, \mathrm{V}}, \lambda_{\mathrm{K}, \mathrm{V}}\right)-h_{\mathrm{K}, \mathrm{V}}}{\theta_{\mathrm{K}, \mathrm{V}}}, \\
\tau_{\mathrm{K}, \mathrm{V}} & =\frac{c}{\exp \left\{\left(V-\overline{V_{\mathrm{K}}}\right) / a\right\}+\exp \left\{-\left(V-\overline{V_{\mathrm{K}}}\right) / b\right\}} .
\end{aligned}
$$

The calcium-gated potassium channel $(\mathrm{K}, \mathrm{Ca})$ is defined as

$$
\begin{aligned}
I_{\mathrm{K}, \mathrm{Ca}} & =g_{\mathrm{K}, \mathrm{Ca}} \overline{g_{\mathrm{K}, \mathrm{Ca}}}\left(V-\overline{V_{\mathrm{K}}}\right) H\left(C, C_{\mathrm{K}, \mathrm{Ca}}, n_{\mathrm{K}, \mathrm{Ca}}\right), \\
\frac{d g_{\mathrm{K}, \mathrm{Ca}}}{d t} & =\frac{\sigma_{\mathrm{act}}\left(V, V_{\mathrm{K}, \mathrm{Ca}}, \kappa_{\mathrm{K}, \mathrm{Ca}}\right)-g_{\mathrm{K}, \mathrm{Ca}}}{\tau_{\mathrm{K}, \mathrm{Ca}}}, \\
\frac{d C_{\mathrm{K}, \mathrm{Ca}}}{d t} & =\frac{C_{\mathrm{K}, \mathrm{Ca}}^{\infty}(V)-C_{\mathrm{K}, \mathrm{Ca}}}{\tau_{\mathrm{K}, \mathrm{Ca}}}, \\
C_{\mathrm{K}, \mathrm{Ca}}^{\infty}(V) & =c \exp \left\{\frac{a-V}{b}\right\} .
\end{aligned}
$$

All parameters are in analogy to the other proteins described before.

The small conductance calcium-gated potassium channel (sK,Ca) is modeled as

$$
\begin{array}{r}
I_{\mathrm{sK}, \mathrm{Ca}}=g_{\mathrm{sK}, \mathrm{Ca}} \overline{g_{\mathrm{sK}, \mathrm{Ca}}}\left(V-\overline{V_{\mathrm{K}}}\right), \\
\frac{d g_{\mathrm{sK}, \mathrm{Ca}}}{d t}=\frac{\sigma_{\mathrm{act}}\left(C, C_{\mathrm{sK}, \mathrm{Ca}}, \kappa_{\mathrm{sK}, \mathrm{Ca}}\right)-g_{\mathrm{sK}, \mathrm{Ca}}}{\tau_{\mathrm{sK}, \mathrm{Ca}}} .
\end{array}
$$

The voltage-gated calcium channels $(\mathrm{Ca}, L / T)$ induce the inflow of calcium. The dominant types in $\beta$-cells resemble $L$ and $T$-type channels. The equations for these channels are in analogy to the voltage-gated sodium channels (A4),

$$
\begin{aligned}
I_{\mathrm{Ca}, L} & =h_{\mathrm{Ca}, L}\left[1-H\left(C, C_{\mathrm{Ca}, L}, n_{\mathrm{Ca}, L}\right)\right] g_{\mathrm{Ca}, L} \overline{g_{\mathrm{Ca}, L}}\left(V-\overline{V_{\mathrm{Ca}}}\right), \\
\frac{d g_{\mathrm{Ca}, L}}{d t} & =\frac{\sigma_{\mathrm{act}}\left(V, V_{\mathrm{Ca}, L}, \kappa_{\mathrm{Ca}, L}\right)-g_{\mathrm{Ca}, L}}{\tau_{\mathrm{Ca}, L}}, \\
\frac{d h_{\mathrm{Ca}, L}}{d t} & =\frac{\sigma_{\text {inact }}\left(V, W_{\mathrm{Ca}, L}, \lambda_{\mathrm{Ca}, L}\right)-h_{\mathrm{Ca}, L}}{\theta_{\mathrm{Ca}, L}} .
\end{aligned}
$$

For $T$-type channels, all $L$ 's in the subscript have to be replaced by $T$. The term $1-H(\cdots)$ appears only for $L$-type channels and has to be replaced by 1 for $T$-type.

The leakage currents in (1) are calculated in the steadystate (s) approximation of all kinetic equations for the open probabilities,

$$
\begin{aligned}
-J_{\mathrm{Na}}= & \rho_{\mathrm{Na}, \mathrm{V}} I_{\mathrm{Na}, \mathrm{V}}^{\mathrm{s}}+2 \rho_{\mathrm{Na}, \mathrm{K}} I_{\mathrm{Na}, \mathrm{K}}^{\mathrm{s}} \alpha_{\mathrm{Na}, \mathrm{K}}+\rho_{\mathrm{NCX}} I_{\mathrm{NCX}}^{\mathrm{s}} \alpha_{\mathrm{NCX}}, \\
-J_{\mathrm{K}}= & \rho_{\mathrm{K}, \mathrm{ATP}} I_{\mathrm{K}, \mathrm{ATP}}^{\mathrm{s}}+\rho_{\mathrm{K}, \mathrm{V}} I_{\mathrm{K}, \mathrm{V}}^{\mathrm{s}}+\rho_{\mathrm{SK}, \mathrm{Ca}} I_{\mathrm{sK}, \mathrm{Ca}}^{\mathrm{s}} \\
& +\rho_{\mathrm{K}, \mathrm{Ca}} I_{\mathrm{K}, \mathrm{Ca}}^{\mathrm{s}}-2 \rho_{\mathrm{Na}, \mathrm{K}} I_{\mathrm{Na}, \mathrm{K}}^{\mathrm{s}}, \\
-J_{\mathrm{Ca}}= & \rho_{\mathrm{Ca}, L} I_{\mathrm{Ca}, L}^{\mathrm{s}}+\rho_{\mathrm{Ca}, T} I_{\mathrm{Ca}, T}^{\mathrm{s},}-z_{\mathrm{Ca}} \rho_{\mathrm{NCX}} I_{\mathrm{NCX}}^{\mathrm{s}} \\
& +\rho_{\mathrm{PMCA}} I_{\mathrm{PMCA}}^{\mathrm{s}},
\end{aligned}
$$

$I_{x}^{\mathrm{s}}$ currents are evaluated based on properties in the resting state $\left\{V_{0}, \gamma_{0}, N_{0}, K_{0}, C_{0}\right\}$, e.g.,

$$
\begin{aligned}
I_{\mathrm{Na}, \mathrm{K}}^{\mathrm{s}}= & \overline{I_{\mathrm{Na}, \mathrm{K}}}\left[1-H\left(K_{0}, K_{\mathrm{Na}, \mathrm{K}}, n_{\mathrm{Na}, \mathrm{K}}\right)\right] H\left(N_{0}, \tilde{K}_{\mathrm{Na}, \mathrm{K}}, \tilde{n}_{\mathrm{Na}, \mathrm{K}}\right), \\
I_{\mathrm{Na}, \mathrm{V}}^{\mathrm{s}}= & \sigma_{\mathrm{act}}\left(V_{0}, V_{\mathrm{Na}, \mathrm{V}}, \kappa_{\mathrm{Na}, \mathrm{V}}\right) \sigma_{\mathrm{inact}}\left(V_{0}, W_{\mathrm{Na}, \mathrm{V}}, \lambda_{\mathrm{Na}, \mathrm{V}}\right) \\
& \times \overline{g_{\mathrm{Na}, \mathrm{V}}}\left(V_{0}-\overline{V_{\mathrm{Na}}}\right) .
\end{aligned}
$$

The functional form of these equations is justified in [13] based on single trans-membrane protein measurements. All parameter values can be found in Tables 3-5 of the Supplemental Material of [13].

\section{APPENDIX B: MAPPING OF THE $\beta$-CELL MODEL TO THE GENERAL MODEL (9)}

Mapping the $\beta$-cell model to the general form (9) is not possible unless some assumptions are considered. In what follows, we explain under which conditions this mapping is possible.

We found that DESyn is also observable in the network of two coupled $\beta$-cells with nondelayed coupling, i.e., $\tau_{\text {gap }}^{\text {ion }} \rightarrow 0 s$ (data not shown). For the nondelayed gap junction current, we have (coupling dynamics are removed)

$$
I_{\text {gap } ; i j}^{\text {ion }}=\overline{g_{\text {gap }}}\left(V_{i}-V_{j}-\tilde{V}_{i j}^{\text {ion }}\right), \quad i, j=1,2, \quad i \neq j .
$$


Thus, the dynamics of gap junction currents are removed. Further, we assume a constant Nernst potential $\tilde{V}_{i j}^{\text {ion }}=\tilde{V}^{\text {ion }}$. Based on this assumption, (1) is simplified to

$$
\begin{aligned}
\frac{d N_{i}}{d t}= & -\frac{\xi}{F}\left(\rho_{\mathrm{Na}, \mathrm{V}} I_{\mathrm{Na}, \mathrm{V} ; i}+2 \alpha_{\mathrm{Na}, \mathrm{K}} \rho_{\mathrm{Na}, \mathrm{K}} I_{\mathrm{Na}, \mathrm{K} ; i}+\alpha_{\mathrm{NCX}} \rho_{\mathrm{NCX}} I_{\mathrm{NCX} ; i}+J_{\mathrm{Na}}+\frac{1}{6} \rho_{\mathrm{gap}} \overline{g_{\text {gap }}}\left(V_{i}-V_{j}\right)-\frac{1}{6} \rho_{\mathrm{gap}} \overline{g_{\text {gap }}} \tilde{V}^{\mathrm{N}}\right), \\
\frac{d K_{i}}{d t}= & -\frac{\xi}{F}\left(\rho_{\mathrm{K}, \mathrm{ATP} ; i} I_{\mathrm{K}, \mathrm{ATP} ; i}+\rho_{\mathrm{K}, \mathrm{V}} I_{\mathrm{K}, \mathrm{V} ; i}-\frac{4}{3} \alpha_{\mathrm{Na}, \mathrm{K}} \rho_{\mathrm{Na}, \mathrm{K}} I_{\mathrm{Na}, \mathrm{K} ; i}\right. \\
& \left.+\rho_{\mathrm{sK}, \mathrm{Ca}} I_{\mathrm{sK}, \mathrm{Ca} ; i}+\rho_{\mathrm{K}, \mathrm{Ca}} I_{\mathrm{K}, \mathrm{Ca}, i}+J_{\mathrm{K}}+\frac{1}{6} \rho_{\mathrm{gap}} \overline{g_{\mathrm{gap}}}\left(V_{i}-V_{j}\right)-\frac{1}{6} \rho_{\mathrm{gap}} \overline{g_{\mathrm{gap}}} \tilde{V}^{\mathrm{K}}\right), \\
\frac{d C_{i}}{d t}= & -\frac{\xi}{z_{\mathrm{Ca}} F\left(1+x_{\mathrm{c} ; i}\right)}\left(\rho_{\mathrm{Ca}, L} I_{\mathrm{Ca}, L ; i}+\rho_{\mathrm{Ca}, T} I_{\mathrm{Ca}, T ; i}+\rho_{\mathrm{PMCA}} I_{\mathrm{PMCA} ; i}\right. \\
& \left.-\frac{1}{3} z_{\mathrm{Ca}} \alpha_{\mathrm{NCX}} \rho_{\mathrm{NCX}} I_{\mathrm{NCX} ; i}+J_{\mathrm{Ca}}+\frac{1}{6} \rho_{\mathrm{gap}} \overline{g_{\mathrm{gap}}}\left(V_{i}-V_{j}\right)-\frac{1}{6} \rho_{\mathrm{gap}} \overline{g_{\mathrm{gap}}} \tilde{V}^{\mathrm{C}}\right), \\
\frac{d V_{i}}{d t}= & -\frac{1}{C_{\mathrm{m}}}\left(\rho_{\mathrm{Na}, \mathrm{K}} I_{\mathrm{Na}, \mathrm{K} ; i}+\rho_{\mathrm{K}, \mathrm{ATP} ; i} I_{\mathrm{K}, \mathrm{ATP} ; i}+\rho_{\mathrm{K}, \mathrm{V}} I_{\mathrm{K}, \mathrm{V} ; i}+\rho_{\mathrm{Na}, \mathrm{V}} I_{\mathrm{Na}, \mathrm{V} ; i}+\rho_{\mathrm{sK}, \mathrm{Ca}} I_{\mathrm{sK}, \mathrm{Ca} ; i}+\rho_{\mathrm{K}, \mathrm{Ca}} I_{\mathrm{K}, \mathrm{Ca} ; i}+\rho_{\mathrm{NCX}} I_{\mathrm{NCX} ; i}\right. \\
& \left.+\rho_{\mathrm{PMCA}} I_{\mathrm{PMCA} ; i}+\rho_{\mathrm{Ca}, L} I_{\mathrm{Ca}, L ; i}+\rho_{\mathrm{Ca}, T} I_{\mathrm{Ca}, T ; i}+J_{\mathrm{Na}}+J_{\mathrm{K}}+J_{\mathrm{Ca}}+\frac{1}{2} \rho_{\mathrm{gap}} \overline{g_{\mathrm{gap}}}\left(V_{i}-V_{j}\right)-\frac{1}{6} \sum_{\text {ion }} \rho_{\mathrm{gap}} \frac{g_{\text {gap }}}{V^{\text {ion }}}\right) .
\end{aligned}
$$

To cast the resting dynamics in the form of (9), we have

$$
X_{j}=\left[N_{j}, K_{j}, C_{j}, V_{j}, g_{\mathrm{Na}, \mathrm{V} ; j}, h_{\mathrm{Na}, \mathrm{V} ; j}, g_{\mathrm{K}, \mathrm{ATP} ; j}, g_{\mathrm{K}, \mathrm{V} ; j}, h_{\mathrm{K}, \mathrm{V} ; j}, g_{\mathrm{K}, \mathrm{Ca} ; j}, C_{\mathrm{K}, \mathrm{Ca} ; j}, g_{\mathrm{sK}, \mathrm{Ca} ; j}, g_{\mathrm{Ca}, L ; j}, h_{\mathrm{Ca}, L ; j}, g_{\mathrm{Ca}, T ; j}, h_{\mathrm{Ca}, T ; j}\right], \quad j=1,2 .
$$

$g_{c}=\rho_{\text {gap }} \overline{g_{\text {gap }}}$ and $D$ is a square matrix of size 16 with all elements equal to 0 except for $D(k, 4)=-\frac{1}{6}, k=1,2,3$, and $D(4,4)=-\frac{1}{2} \cdot \rho_{\mathrm{K}, \mathrm{ATP} ; j}$ can be considered as the bifurcation parameter $\kappa_{j}$. Finally, the vector $F$ contains all the remaining terms in (B2) excluding the coupling terms.

Note that the simplified $\beta$-cell model in the representation (9) can still show bursts of spikes. However, for the further simplification and reduction of (9) to the normal form, we only focus on the dynamics of spikes. As explained before, DESyn is due to structural changes in the behavior of spikes from asynchronous oscillations to death. In the framework of the normal form, these changes are related to the onset of Hopf bifurcation.

\section{APPENDIX C: INTERMEDIATE STEPS IN THE DERIVATION OF THE NORMAL FORM}

To calculate $G_{j}(t, \tau)$ and $H_{j}(t, \tau)$ in (15), substituting (15) in (14) and setting equal the coefficients of $\varepsilon$, we obtain

$$
G_{j}(t, \tau)=A_{j}(\tau) U e^{i \omega_{0} t}+\bar{A}_{j}(\tau) \bar{U} e^{-i \omega_{0} t},
$$

where $U$ (with the complex conjugate $\bar{U}$ ) is the right eigenvector of $L_{0}$ at $i \omega_{0}$. Setting equal the coefficients of $\varepsilon^{2}$ gives

$$
\begin{aligned}
H_{j}(t, \tau)= & V_{+} A_{j}^{2}(\tau) e^{2 i \omega_{0} t}+V_{-} \bar{A}_{j}^{2}(\tau) e^{-2 i \omega_{0} t} \\
& +V_{0}\left|A_{j}\right|^{2}+S_{0} G_{j}(t, \tau)
\end{aligned}
$$

where $V_{+}, V_{-}$, and $V_{0}$ are defined in (18), and $S_{0}$ is constant. Finally, setting equal the coefficients of $\varepsilon^{3}$ results in the dynamics of the complex oscillation amplitude $A_{j}$ as (16).

In investigating synchronization, coupling terms play a significant role. The coupling terms $g_{c} D\left(X_{k}-X_{j}\right), j, k=$ $1,2, j \neq k$ are related to the dissipation term in the normal form (i.e., the last two terms in each $\dot{r}_{j}, j=1,2$ ) via time scalings $\tau=\varepsilon^{2} t$ and $\hat{\tau}=\mu_{1} a \tau$, coordinate transformations: $Y_{j}=X_{j}-\bar{X}_{j}$, (15) with (16), (C1), (C2), and (19), and the changes of parameters (17), (18), and (21).
[1] A. S. Pikovsky, M. G. Rosenblum, and J. Kurths, Synchronization: A Universal Concept in Nonlinear Sciences (Cambridge University Press, New York, 2001).

[2] P. A. Tass, Phase Resetting in Medicine and Biology. Stochastic Modelling and Data Analysis (Springer-Verlag, Berlin, 1999).

[3] S. H. Strogatz, J. Math. Biol. 25, 327 (1987).
[4] A. Goldbeter and M. J. Berridge, Biochemical Oscillations and Cellular Rhythms (Cambridge University Press, Cambridge, 1997), p. 31.

[5] R. K. Benninger, M. Zhang, W. S. Head, L. S. Satin, and D. W. Piston, Biophys. J. 95, 5048 (2008).

[6] W. S. Head, M. L. Orseth, C. S. Nunemaker, L. S. Satin, D. W. Piston, and R. K. Benninger, Diabetes 61, 1700 (2012). 
[7] L. M. Nguyen, M. Pozzoli, T. H. Hraha, and R. K. Benninger Diabetes 63, 1685 (2014).

[8] R. K. Benninger and D. W. Piston, Trends Endocrin. Met. 25, 399 (2014).

[9] P. Rorsman and E. Renström, Diabetologia 46, 1029 (2003).

[10] T. R. Chay and J. Keizer, J. Biophys. 42, 181 (1983).

[11] M. Zhang, P. Goforth, R. Bertram, A. Sherman, and L. Satin, Biophys. J. 84, 2852 (2003).

[12] M. A. Ravier, M. Güldenagel, A. Charollais, A. Gjinovci, D. Caille, G. Shl, C. B. Wollheim, K. Willecke, J.-C. Henquin, and P. Meda, Diabetes 54, 1798 (2005).

[13] M. E. Meyer-Hermann, Biophys. J. 93, 2952 (2007).

[14] M. E. Meyer-Hermann and R. K. Benninger, HFSP J. 4, 61 (2010).

[15] F. M. Ashcroft, D. E. Harrison, and S. J. H. Ashcroft, Nature (London) 312, 446 (1984).

[16] S. Göpel, T. Kanno, S. Barg, J. Galvanovskis, and P. Rorsman, J. Physiol. 521, 717 (1999).

[17] T. Jüngling, I. Fischer, E. Schöll, and W. Just, Phys. Rev. Lett. 115, 194101 (2015).

[18] B. Doiron, J. Rinzel, and A. Reyes, Phys. Rev. E 74, 030903 (2006).

[19] J. Aguirre, R. Sevilla-Escoboza, R. Gutiérrez, D. Papo, and J. M. Buldú, Phys. Rev. Lett. 112, 248701 (2014).

[20] C. Cherubini, S. Filippi, A. Gizzi, and A. Loppini, Phys. Rev. E 92, 042702 (2015).

[21] Y. Braiman, W. L. Ditto, K. Wiesenfeld, and M. L. Spano, Phys. Lett. A 206, 54 (1995).

[22] L. V. Gambuzza, J. Gómez-Gardeñes, and M. Frasca, Sci. Rep. 6, 24915 (2016).
[23] R. Vicente, L. L. Gollo, C. R. Mirasso, I. Fischer, and G. Pipa, Proc. Natl. Acad. Sci. (USA) 105, 17157 (2008).

[24] R. Banerjee, D. Ghosh, E. Padmanaban, R. Ramaswamy, L. M. Pecora, and S. K. Dana, Phys. Rev. E 85, 027201 (2012).

[25] R. Gutiérrez, R. Sevilla-Escoboza, P. Piedrahita, C. Finke, U. Feudel, J. M. Buldú, G. Huerta-Cuellar, R. Jaimes-Reátegui, Y. Moreno, and S. Boccaletti, Phys. Rev. E 88, 052908 (2013).

[26] L. V. Gambuzza, M. Frasca, L. Fortuna, and S. Boccaletti, Phys. Rev. E 93, 042203 (2016).

[27] A. Bergner, M. Frasca, G. Sciuto, A. Buscarino, E. J. Ngamga, L. Fortuna, and J. Kurths, Phys. Rev. E 85, 026208 (2012).

[28] L. V. Gambuzza, A. Cardillo, A. Fiasconaro, L. Fortuna, J. Gómez-Gardeñes, and M. Frasca, Chaos 23, 043103 (2013).

[29] L. V. Gambuzza, M. Frasca, and J. Gómez-Gardeñes, Europhys. Lett. 110, 20010 (2015).

[30] R. Rosenblum, A. Pikovsky, J. Kurths, C. Schäfer, and P. A. Tass, Phase Synchronization: From Theory to Data Analysis (Elsevier Science, North-Holland, 2001), pp. 279-321.

[31] X. Zhang, A. Daou, T. M. Truong, R. Bertram, and M. G. Roper, Am. J. Physiol. 301, E742 (2011).

[32] M. Zarkovic and J. C. Henquin, Am. J. Phys. 287, E340 (2004).

[33] P. C. Matthews, R. E. Mirollo, and S. H. Strogatz, Physica D 52, 293 (1991).

[34] R. E. Mirollo and S. H. Strogatz, J. Stat. Phys. 60, 245 (1990).

[35] Y. Kuramoto, Chemical Oscillations, Waves, and Turbulence (Springer-Verlag, Berlin, 1984).

[36] D. Aronson, G. Ermentrout, and N. Kopell, Physica D 41, 403 (1990).

[37] G. De Vries, A. Sherman, and H.-R. Zhu, B. Math. Biol. 60, 1167 (1998). 